DR. MARJO RENKO (Orcid ID : 0000-0003-0507-4773)

Article type : Brief Report

\title{
Change in respiratory syncytial virus seasonality in Finland
}

\section{Marjo Renko ${ }^{1,2}$ and Terhi Tapiainen 2,3}

1 Department of Paediatrics, University of Eastern Finland and Kuopio University Hospital, Kuopio, Finland

2 PEDEGO Research Unit, University of Oulu, Oulu, Finland

3 Department of Children and Adolescents, Oulu University Hospital, Oulu, Finland

Corresponding author:

Marjo Renko, prof

Paediatrician, specialist in paediatric infectious diseases

University of Eastern Finland and Kuopio University Hospital

Building 6, 5th floor,

Box 100

70029 Kuopio, Finland

marjo.renko@uef.fi

044-717 6778

RSV (respiratory syncytial virus) is the most frequent cause of lower respiratory tract infection in infants, with a hospitalization rate up to 35 per 1,000 children younger than one year (1). RSV causes bronchiolitis, wheezing bronchitis and bronchopneumonia demanding respiratory support. Altogether $10 \%$ of RSV positive hospitalized children need intensive care (2). RSV infection can be severe particularly in preterm infants and infants with severe congenital heart defects. In high-risk children, monthly prophylaxis with monoclonal antibody decreases the severity of

This article has been accepted for publication and undergone full peer review but has not been through the copyediting, typesetting, pagination and proofreading process, which may lead to differences between this version and the Version of Record. Please cite this article as doi: $\underline{10.1111 / \text { apa. } 14983}$

This article is protected by copyright. All rights reserved 
RSV illness during the epidemics. Anticipating the timing of the RSV season is important for cost-efficient use of prophylaxis with monoclonal antibody. In recent years, data on RSV epidemiology have increased through better availability of fast PCR methods. Many countries have included detection of RSV to influenza surveillance system.

Epidemiology of RSV shows major differences between countries but consistency within countries in time $(1,3)$. In most countries, the start, end and peak of the epidemic differs only 1-3 weeks between the seasons (3). Interestingly, in Nordic countries, biennial epidemiological pattern is typical with a large epidemic every two years alternating with a minor one. This kind of biennial seasonality has been reported, in addition to Nordic countries, in Croatia and Germany $(1,4)$.

In Finland, the biennial RSV epidemic pattern has been particularly clear, as the minor peak in late spring and early summer have almost conjoined with the major winter peak later the same year, causing peculiar epidemiological pattern with every second winter without any or very few RSV cases (5). During the last decade, we recognized in clinical practice, however, that anticipating the next RSV epidemic season and the optimal timing of prophylaxis with monoclonal antibody became increasingly difficult. We suspected that the epidemiological pattern was changing. As RSV seasonality has been described to be constant in time within countries, we aimed to document the suspected change in RSV epidemiology in Finland. To analyse RSV seasonality in Finland, we used the numbers of monthly RSV positive respiratory samples in all ages in 1995-2018.

The numbers of RSV positive samples were retrieved from the National Infectious Diseases Register (NIDR), a comprehensive database of the National Institute for Health and Welfare, Helsinki, Finland comprising all RSV positive samples from the microbiological laboratories in Finland since 1995. The anonymized NIDR registry data, including the date of sampling, the age group and hospital district, is freely available online for data retrieval for clinicians and researchers. To describe the seasonality of RSV in Finland, we retrieved the total monthly numbers of RSV positive samples in Finland from the NIDR, including all hospital districts and age groups. We plotted the monthly numbers of RSV positive samples to describe the epidemic peaks from January 1995 to December 2018.

In odd years in 1990's and early 00's, a large epidemic starting in November followed rapidly after earlier small spring epidemic in May (Figure). In even years after the 
major epidemic peak, RSV cases were virtually absent. In 2008 epidemiological pattern changed in Finland. Since 2008, the RSV epidemics started to occur regularly every winter, peaking in February to May (Figure). Biennial pattern was still seen in the number of RSV cases but the intervals between the peaks level out (Figure). The observed higher absolute number of annual RSV cases in recent years most likely reflects the more easily available PCR-based diagnostics.

Our main finding was that RSV seasonality changed within a country in time. The reason for the observed change in RSV epidemiology in Finland is not known. Climate and temperature may influence the epidemiology of RSV (2). Influenza H1N1 in 2009 has been suggested to have affected RSV epidemiology. The interplay between bacteria and viruses may change viral epidemiology. Universal pneumococcal conjugate vaccination in infants was started in Finland in 2010. The shift of the RSV epidemic peak, however, seems to have occurred in Finland already in 2008. To our knowledge, similar changes have not yet been reported from other countries.

In addition to changing RSV epidemiology, we noted that active viral diagnostics in recent years showed a markedly increased number of annual RSV cases. Influenza testing has changed during the years of this study and PCR based methods have replaced antigen testing. Most paediatric clinics in Finland perform active viral diagnostics throughout the year. Recently, active RSV diagnostics has increased among adults and the elderly, in particular accompanied with influenza surveillance. It seems unlikely, however, that the clear change in the seasonality of RSV is due to changes in laboratory methods and clinical practice. Strength of our study is that we had a comprehensive national registry data comprising all microbiologically confirmed RSV cases for 25 years available. We lacked however, the total number of obtained samples in the national registry data. Thus, the absolute length of the epidemic is difficult to measure in the present study as compared to other studies (1).

In conclusion, our study shows that RSV epidemiological pattern can change in time within one country. Documented change in RSV epidemiology demonstrates the importance of ongoing surveillance of RSV epidemiology to timely implement preventing strategies.

\section{Funding}

This work was completed without any specific funding. 


\section{Conflicts of interest}

No conflicts of interest.

\section{References:}

1. Broberg EK, Waris M, Johansen K, Snacken R, Penttinen $P$, European Influenza Surveillance Network. Seasonality and geographical spread of RSV epidemics in 15 European countries. Euro Surveill 2018;23(5).

2. Sirimi N, Miligkos M, Koutouzi M, Petridou E, Siahanidou T, Michos A. Respiratory Syncytial Virus Activity and Climate Parameters During a 12-Year Period. J Med Virol 88:931-937, 2016.

3. Obando-Pacheco P, Justicia-Grande AJ, Rivero-Calle I, Rodríguez-Tenreiro C, Sly P, Ramilo O, et al. Respiratory Syncytial Virus Seasonality: A Global Overview. J Infect Dis 2018;217:1356-64.

4. Jepsen MT, Trebbien R, Emborg HD, Krause TG, Schønning K, Voldstedlund $M$, et al. Incidence and seasonality of respiratory syncytial virus hospitalisations in young children in Denmark, 2010 to 2015. Euro Surveill. 2018;23(3).

5. Waris M. Pattern of respiratory syncytial virus epidemics in Finland: two-year cycles with alternating prevalence of groups A and B. J Infect Dis. 1991;163(3):464-9.

\section{Figure legend.}

Total number of monthly RSV cases in all age groups in National Infectious Diseases Registry in Finland in 1995-2018. Number of RSV cases 1995-2006 (upper line): every odd year a small spring epidemic was followed by a large epidemic peaking around December. After 2007 (lower line) the epidemics started to peak every year around March. Time in months between RSV epidemic peaks is presented in the figure. 


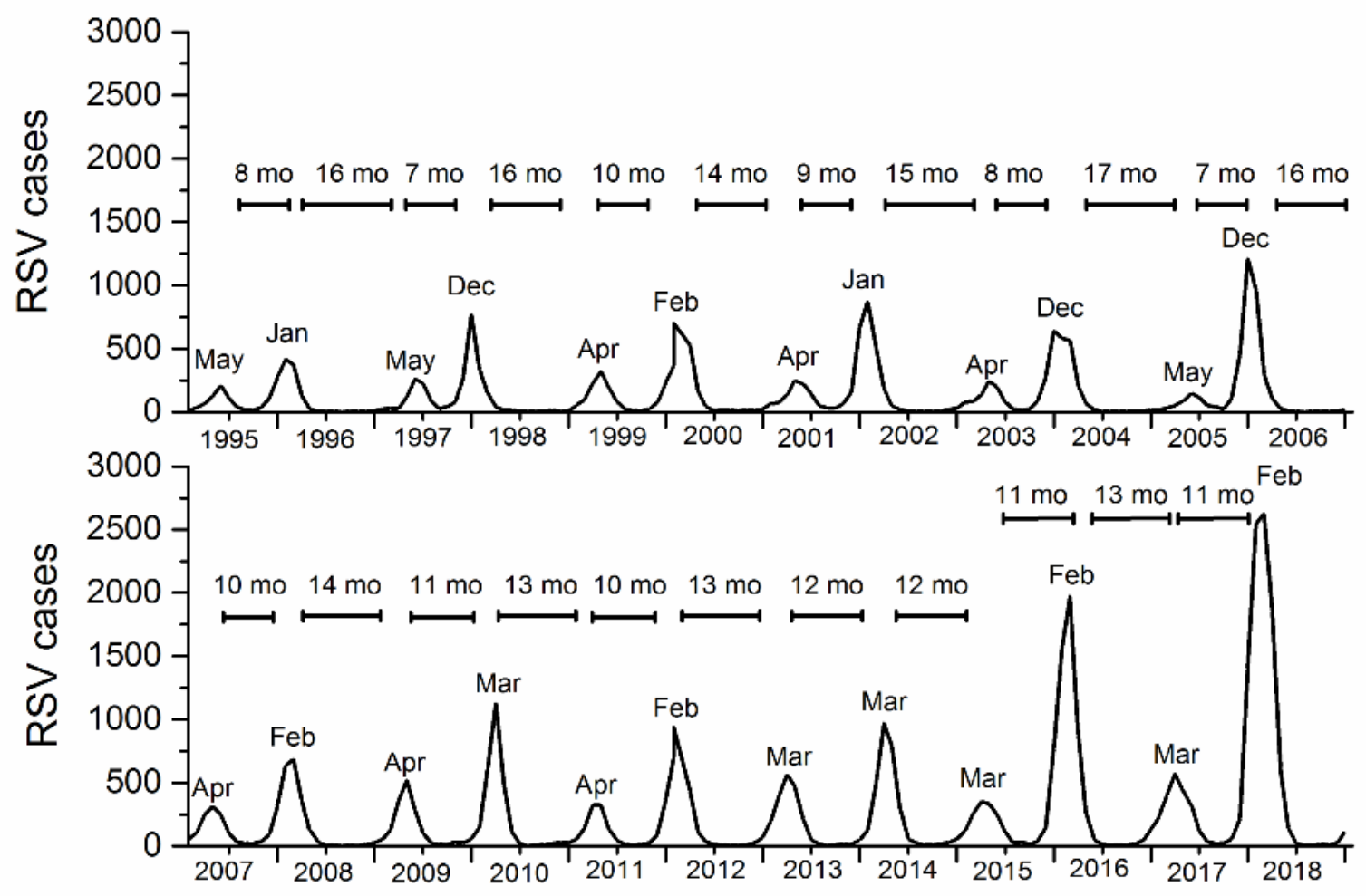

apa_14983_f1.tif

This article is protected by copyright. All rights reserved 